\title{
Functional validation of TERT and TERC variants of uncertain significance in patients with short telomere syndromes
}

\author{
Alejandro Ferrer (10) ${ }^{1,2}$, Abhishek A. Mangaonkar ${ }^{3}$, Susanna Stroik ${ }^{4,9}$, Michael T. Zimmermann (1) , Ashley N. Sigafoos ${ }^{6}$, \\ Patrick S. Kamath7, Douglas A. Simonetto ${ }^{7}$, Mark E. Wylam ${ }^{8}$, Eva M. Carmona ${ }^{8}$, Konstantinos N. Lazaridis ${ }^{1,7}$, \\ Steve Peters ${ }^{8}$, Keith Stewart ${ }^{1,2}$, Eric W. Klee ${ }^{1,2}$, Eric A. Hendrickson $\mathbb{1 0}^{4}$ and Mrinal M. Patnaik $\mathbb{B}^{3}$
}

\section{Dear Editor,}

Accelerated shortening of telomeres can induce premature cell senescence that can clinically manifest as bone marrow failure (BMF), idiopathic pulmonary fibrosis (IPF), cryptogenic cirrhosis, nodular regenerative hyperplasia, vascular malformations, immunodeficiency, and structural brain abnormalities, all of which are included under the umbrella term of short telomere syndromes $(\mathrm{STSs})^{1}$. Two main criteria used to diagnose STS include the documentation of shortened telomere lengths (TLs) by a Clinical Laboratory Improvement Amendments (CLIA)-certified flowFISH (fluorescence in situ hybridization) assay ${ }^{2}$ and the presence of pathogenic variants in genes related to telomere maintenance identified through next-generation sequencing (NGS). These genes include, but are not limited to, $h T E R T$ (human telomerase reverse transcriptase) and hTERC (human telomerase RNA component), the two main components of the telomerase holoenzyme complex that is responsible for creating new telomeric DNA (Supplementary Fig. 1) ${ }^{1}$.

In our experience, a pathogenic variant in the coding sequence of a telomere-associated gene can be identified in only $40 \%$ of STS cases using current sequencing approaches, with the remaining cases either having no identifiable variant or possessing a variant(s) of uncertain significance (VUS) ${ }^{1,3}$. In addition, in some of these cases, the TL values were not conclusively shortened (i.e., not $<$

\footnotetext{
Correspondence: Eric A. Hendrickson (hendr064@umn.edu) or

Mrinal M. Patnaik (patnaik.mrinal@mayo.edu)

${ }^{1}$ Center for Individualized Medicine, Mayo Clinic, Rochester, MN, USA

${ }^{2}$ Department of Health Sciences Research, Mayo Clinic, Rochester, MN, USA

Full list of author information is available at the end of the article

These authors contributed equally: Alejandro Ferrer, Abhishek A. Mangaonkar
}

first percentile in lymphocytes and/or granulocytes documented by a CLIA-certified FlowFISH assay) complicating the diagnosis. At Mayo Clinic, we established a dedicated BMF clinic in collaboration with the center for Individualized Medicine and the division of Hematology, so as to leverage the latest NGS technologies and functional assays to assist patients with unexplained BMF syndromes, including STS-related marrow failure ${ }^{3,4}$. We have developed a systematic algorithmic approach to assess STS patients that includes TL testing by flowFISH and genomic assessment of STS-related gene mutations using an in-house designed targeted NGS panel (Supplementary Table 1). Through this effort, we have identified 32 patients with an STS phenotype and TLs at or below the tenth percentile. Twenty-two patients $(69 \%)$ did not have detectable pathogenic variants (in spite of a clinical phenotype) or were found to carry a VUS in STS-related genes (ten patients; $31 \%$ ). In the latter group, the VUS were located in hTERT (four variants), hTERC (one patient), RTEL1 (regulator of telomere elongation helicase 1; two variants), TINF2 (TERF1-interacting nuclear factor 2; two variants), and NAF1 (nuclear assembly factor 1 ribonucleoprotein; one variant). Patients with $h T E R T$ VUS (Table 1 and Supplemental Fig. 2) were selected for three-dimensional (3D) computational modeling and functional interrogation, largely due to the availability of a well-described functional assays, namely the telomerase repeat amplification protocol (TRAP), which can be used to test telomerase activity (Supplemental Methods) ${ }^{5,6}$.

All patients (Table 1) had BMF with additional STS features (IPF in patients 2 and 5, and premature graying of hair in patients 1 and 2). FlowFISH testing indicated a TL below the first centile in both lymphocytes and 


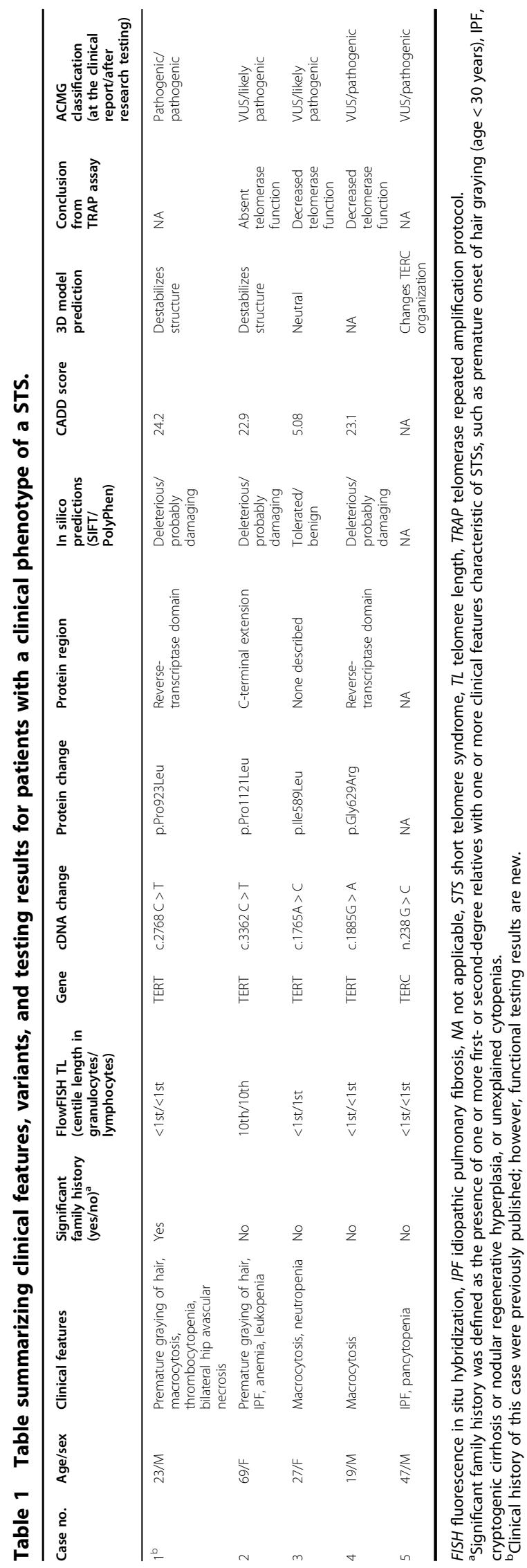

granulocytes in all patients, except in patient 2 were the TL was at the tenth centile range (Fig. 1a). NGS testing uncovered $h T E R T$ variants in patients $1-4$ and a $h T E R C$ variant in patient 5 . To increase our interpretive resolution for $h T E R T$ variants, we created a computational 3D protein model of the human telomerase complex that we used to assess the intra- and inter-molecular interactions within the complex. Our current model includes all amino acids of hTERT and a contiguous section of hTERC with the bound DNA heteroduplex. This model was generated using homology-based methods $\mathrm{s}^{7,8}$ and the experimentally solved structure of TERT from Tetrahymena thermophila [PDB 6d6 $\left.{ }^{9}\right]$. The human Reverse Transcriptase thumb domain of telomerase was experimentally solved $\left[5 \mathrm{ugw}^{10}\right]$ and added to our model using threading. Genomic variants were assessed in their 3D context using FoldX ${ }^{11}$. Recent model fitting to a cryogenic electron microscopy map of human telomerase core particle was published ${ }^{12}$ and was used here in comparison to our current model of full-length TERT.

When indicated, we used our 3D model to suggest mechanisms of pathogenicity for these variants as follows ${ }^{1}$ : hTERT c.2768 C > T; p.Pro923Leu (patient 1) is located near the helix required for oligomerization and is adjacent to p.Arg901 and p.Lys902-sites where bona fide loss-of-function mutations have been described (Fig. 1b) ${ }^{2}$. hTERT c.3362 C > T; p.Pro1121Leu (patient 2) is present at the end of the reverse-transcriptase thumb domain at a position where the peptide chain crosses back against residues 999-1003. Further, hTERC wraps around one side of this thumb and the DNA winds across the other side, so that any changes to the internal organization/ arrangement of this thumb may significantly alter function (Fig. 1b) ${ }^{3}$. The third variant $h T E R T$ c,1765A $>$ C; p.Ile589Leu (patient 3) affects a residue located in the helix near the hTERC interface possibly affecting stability of this interaction (Fig. 1b) ${ }^{4}$. The fourth variant, $h T E R T$ c.1885G > A; p.Gly629Arg is located in a loop between the DNA strand of the heteroduplex, and Lys626 and Arg631, both of which make contact with the DNA strand (Fig. 1b).

In addition to this information, further functional testing was pursued for these variants with the exception of hTERT c.2768 C > T; p.Pro923Leu (Patient 1), where we considered the evidence already available, including a published functional report, to be strong enough to consider this variant as being pathogenic ${ }^{13}$ (more information in ref. ${ }^{3}$ ). For the remaining patients with $h T E R T$ variants, we evaluated their telomerase activity using the TRAP assay ${ }^{5}$. TRAP semi-quantitatively measures the capacity to elongate a telomere-imitating oligonucleotide via hTERT processing in a patient's samples over several amplification cycles, akin to a quantitative PCR reaction. The commercially available TRAPeze Telomerase Detection Kit (Supplemental Methods) was employed in peripheral 

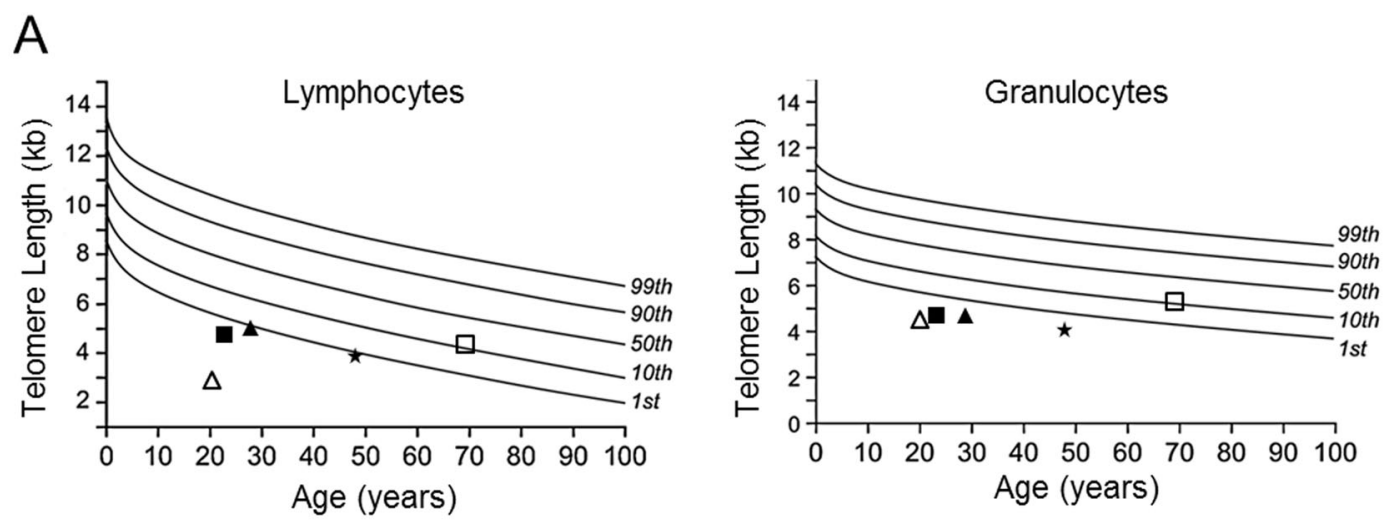

- Patient 1 aPatient $2 \Delta$ Patient $3 \Delta$ Patient $4 \star$ Patient 5
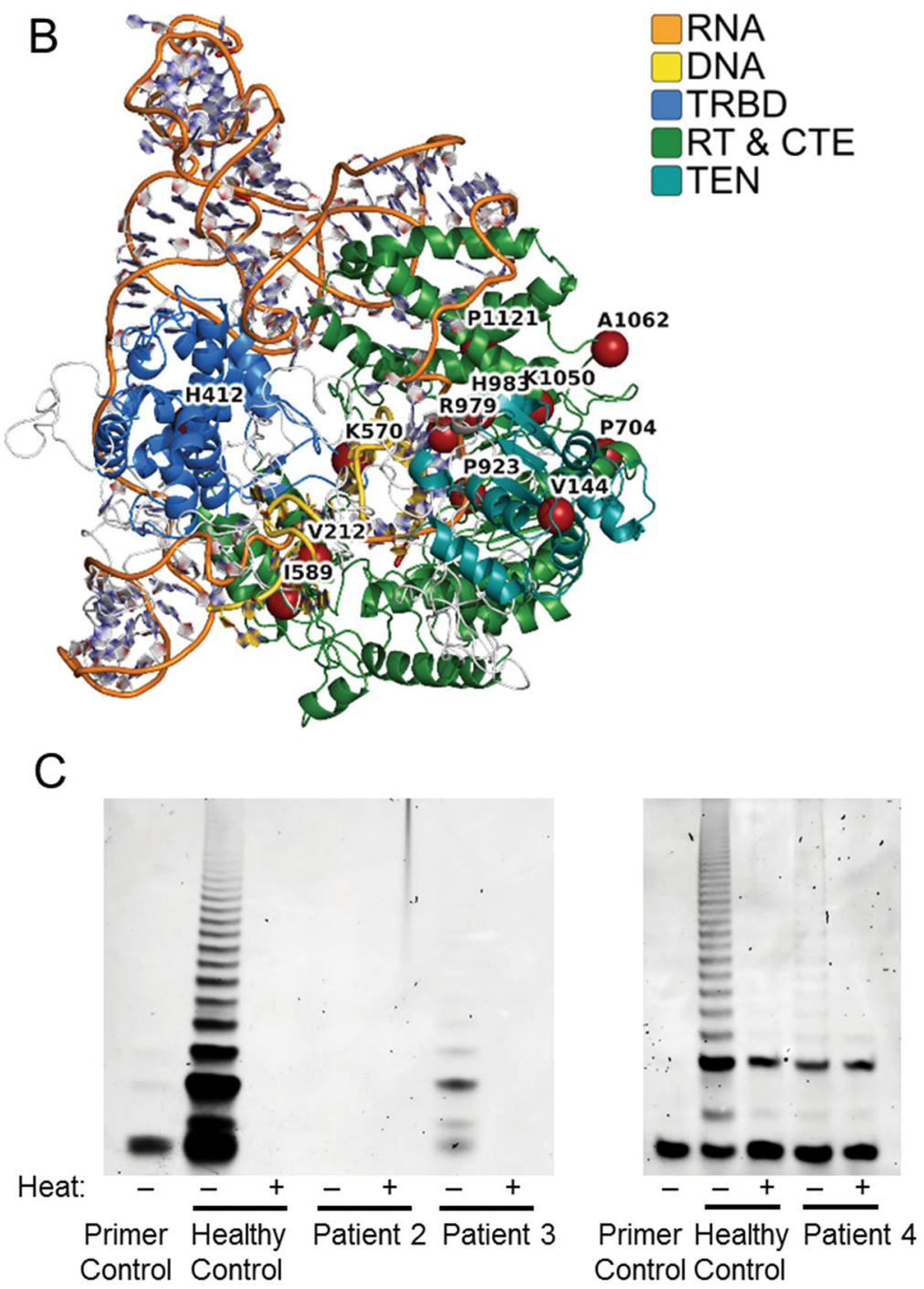

Fig. 1 (See legend on next page.) 
(see figure on previous page)

Fig. 1 Functional validation of variants of uncertain significance in $h T E R T / h T E R C$. a Mean telomere length in lymphocytes and granulocytes from blood samples measured by flowFISH. All patients presented clinical signs of STS and genetic variants in the holozenzyme telomerase. While most patients $(1,3,4$, and 5) presented clearly shortened telomeres, patient 2 showed fringe values closer to the 10th centile. $\mathbf{b}$ Recent studies have resolved human telomerase core components, which can be combined with additional data to generate a more specific and comprehensive model for interpreting the effects of telomerase variation. We used molecular modeling to develop a finer resolution of interpretation for telomerase variants identified through clinical genomics sequencing. The nucleotide backbones of RNA and DNA are colored in orange and gold, respectively, and each protein domain is colored distinctly with the intervening loops demarcated in white. Our current model of human telomeres consists of all amino acids of hTERT and a large contiguous section of hTERC. This model was generated based off of homology relationships and energetic refinement. The 3D relationships among amino acids of TERT and nucleotides of TERC enable us to make specific predictions for the impact and effects of genomic variants. c Blood protein extracts from patients carrying TERT variants were tested using the TRAP assay for telomerase activity. In comparison to healthy individuals used as controls (second lane in each panel), the results indicated absent (patient 2) or diminished (patients 3 and 4) telomerase activity in all samples tested, suggesting pathogenicity of the variants.

blood mononuclear cells from patients $2-4$ and the results indicated decreased telomerase activity in all samples compared to age-matched patient controls (Fig. 1c). This data, together with our 3D-prediction models, allowed us to re-classify these variants as pathogenic or likely pathogenic (Table 1$)$.

The $h T E R C$ VUS in patient $5(\mathrm{n} .238 \mathrm{G}>\mathrm{C}$ ) was located in the stem loop in the CD4/5 region and our 3D model analysis indicated a likely reorganization of the stem loop as a consequence of this change, thereby altering the secondary structure of the RNA. Additionally, a previous report had indicated decreased telomerase activity in patients carrying this variant when tested by the TRAP assay $^{14}$. We felt that given the clinical context, this information was sufficient enough to classify this variant as pathogenic without the need for additional testing.

In conclusion, we demonstrate the importance and feasibility of using 3D molecular modeling and functional assays to classify variants identified in the $h T E R T$ holoenzyme. By using these methods, we were able to provisionally re-classify five VUS identified in patients with STS, an important step forward in mutational nomenclature. Importantly, pathogenic variants in hTERT/hTERC comprise $52 \%$ of STS-related mutations described in the Human Gene Mutation Database (https://portal.biobase-international.com/hgmd/). For the remaining $48 \%$ of mutations that encompass several genes involved in TL regulation, there currently are no reliable functional assays (e.g., assessment of RTEL1 abnormalities by $\mathrm{T}$-circle detection demonstrates considerable variability). Thus, variants identified in our clinic impacting RTEL1, TINF2, and NAF1 still remain unclassifiable and their causal impact on TL in these patients remains unknown. TRAP assays also demonstrate inherent variabilities between tissue samplings and between individuals within the same age range, limiting the routine implementation of these assays in clinic. The development of cell line-based assays utilizing genetic engineering of variants and assessing their impact on TL could be one potential way of overcoming these limitations. In summary, while we were able to assess the pathogenicity of $h T E R T / h T E R C$ variants using 3D modeling and the TRAP assay, a lot more work is needed to help develop accurate in silico approaches and functional testing for variants in other genes regulating TL.

\section{Acknowledgements}

We thank the patients and their families for participating in this study. This research was funded internally by the Center for Individualized Medicine at Mayo Clinic.

\section{Author details}

${ }^{1}$ Center for Individualized Medicine, Mayo Clinic, Rochester, MN, USA. ${ }^{2}$ Department of Health Sciences Research, Mayo Clinic, Rochester, MN, USA. ${ }^{3}$ Department of Hematology, Mayo Clinic, Rochester, MN, USA. ${ }^{4}$ Department of Biochemistry, Molecular Biology and Biophysics, University of Minnesota, Minneapolis, MN, USA. ${ }^{5}$ Genomic Sciences and Precision Medicine Center, Medical College of Wisconsin, Wauwatosa, USA. ${ }^{6}$ Department of Oncology, Mayo Clinic, Rochester, MN, USA. ${ }^{7}$ Department of Gastroenterology, Mayo Clinic, Rochester, MN, USA. ${ }^{8}$ Pulmonary and Critical Care Medicine, Mayo Clinic, Rochester, MN, USA. ${ }^{9}$ Present address: Lineberger Comprehensive Cancer Center, University of North Carolina at Chapel Hill, Chapel, Hill, NC, USA

\section{Conflicts of interest}

The authors declare that they have no conflicts of interest.

\section{Publisher's note}

Springer Nature remains neutral with regard to jurisdictional claims in published maps and institutional affiliations.

Supplementary Information accompanies this paper at (https://doi.org/ 10.1038/s41408-020-00386-z).

Received: 6 August 2020 Revised: 8 September 2020 Accepted: 29 October 2020

Published online: 17 November 2020

\section{References}

1. Mangaonkar, A. A. \& Patnaik, M. M. Short telomere syndromes in clinical practice: bridging bench and bedside. Mayo Clin. Proc. 93, 904-16 (2018).

2. Alder, J. K. et al. Diagnostic utility of telomere length testing in a hospitalbased setting. Proc. Natl Acad. Sci. USA 115, E2358-E2365 (2018).

3. Mangaonkar, A. A. et al. Clinical applications and utility of a precision medicine approach for patients with unexplained cytopenias. Mayo Clin. Proc. 94, 1753-1768 (2019)

4. Mangaonkar, A. A. et al. Clinical correlates and treatment outcomes for patients with short telomere syndromes. Mayo Clin. Proc. 93, 834-839 (2018).

5. Skvortsov, D. A., Zvereva, M. E., Shpanchenko, O. V. \& Dontsova, O. A. Assays for detection of telomerase activity. Acta Nat. 3, 48-68 (2011).

6. Zhang, Z., Hu, Q. \& Zhao, Y. Analysis of telomere-homologous DNA with different conformations using $2 \mathrm{D}$ agarose electrophoresis and in-gel hybridization. Methods Mol. Biol. 1587, 197-204 (2017). 
7. Kallberg, M. et al. Template-based protein structure modeling using the RaptorX web server. Nat. Protoc. 7, 1511-1522 (2012).

8. Yang, J. et al. The I-TASSER Suite: protein structure and function prediction. Nat. Methods 12, 7-8 (2015).

9. Jiang, J. et al. Structure of telomerase with telomeric DNA. Cell 173 1179-1190. e13 (2018).

10. Hoffman, H., Rice, C. \& Skordalakes, E. Structural analysis reveals the deleterious effects of telomerase mutations in bone marrow failure syndromes. J. Biol. Chem. 292, 4593-4601 (2017).
11. Schymkowitz, J. et al. The FoldX web server: an online force field. Nucleic Acids Res. 33, W382-W388 (2005).

12. Nguyen, T. H. D. et al. Cryo-EM structure of substrate-bound human telomerase holoenzyme. Nature 557, 190-195 (2018).

13. Gansner, J. M., Rosas, I. O. \& Ebert, B. L. Pulmonary fibrosis, bone marrow failure, and telomerase mutation. N. Engl. J. Med. 366, 1551-1553 (2012).

14. Junko, T. et al. Identification and functional characterization of novel telomerase variant alleles in Japanese patients with bone-marrow failure syndromes. Blood Cells Mol. Dis. 40, 185-191 (2008). 